\title{
A TWENTY-ONE-YEAR REVIEW OF A CASE OF CONGENITAL INDIFFERENCE TO PAIN
}

\author{
R. P. Drummond, Strathfield, Australia, and G. K. Rose, Oswestry, England
}

From the Robert Jones and Agnes Hunt Orthopaedic Hospital, Oswestry

\begin{abstract}
A boy aged three with indifference to pain was followed up until his death from amyloid disease some twenty-one years later. A full necropsy was done and the neuropathology suggested a sensory neuropathy.
\end{abstract}

This case of congenital indifference to pain was first published in the Journal of Bone and Joint Surgery by G. K. Rose in 1953; the patient's age at the time of diagnosis was three years. This paper provides a twenty-oneyears' follow-up until the patient's death at the age of twenty-five, illustrates the progress of the various characteristics of the syndrome and reports on the findings at the necropsy. The development of large cystic lesions associated with the neuropathic joints and a traumatic microstoma are noted as additional features.

The first indication of the presence of insensitive skin,

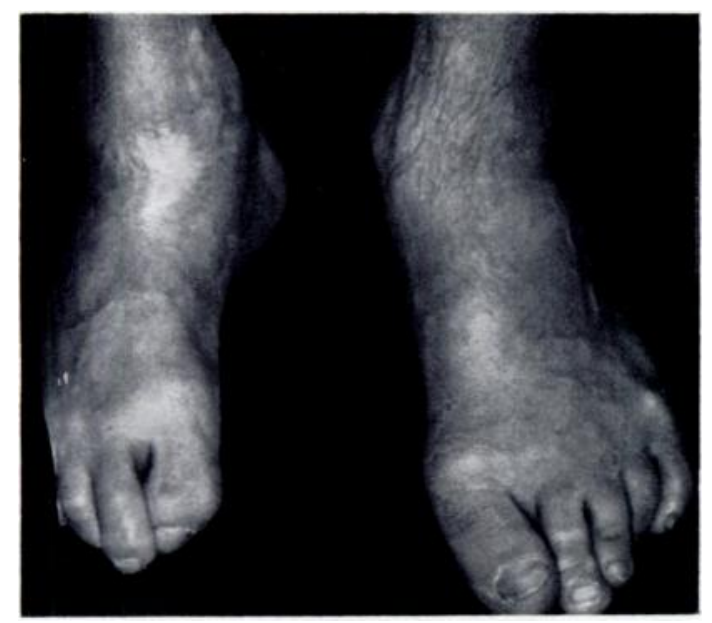

FIG. 1

Wasting and scarring of the right foot at 25 years.

which led to the diagnosis in this patient, was the rapid onset of plaster sores and skin ulceration while he was being treated for bow legs and intoeing at the age of two years. The sores healed when the plasters were removed. The diagnosis was confirmed at three years while the child was being observed in hospital for treatment of osteochondritis of the right talus. The patient painlessly picked skin and subcutaneous tissue from his hands and feet and was indifferent to being suspended by the hair. Examination by a neurologist at this time excluded other causes of loss of pain sensibility, such as syringomyelia.

Immobilisation of a sprained ankle in a below-knee plaster led to popliteal artery occlusion, reported in 1953. A plastic toy was pushed inside the plaster, compressing the artery. Despite lumbar sympathetic block the pulps of the lateral four toes became black and separated; skin on the dorsum of this foot also sloughed. There was no active muscle action at the ankle or toes for five months.

At twenty-five years the marked wasting of the calf muscles and the scarring of the toe pulps and dorsum of the right foot were explained by this early ischaemic episode (Fig. 1).

Indifference to pain-The usual progression of the pain indifference is to gradual recovery of normal pain sensibility. In this patient some pain sensibility was first noticed at six years, and by eleven years no loss of sensation to pin prick could be found. By fourteen a laceration of the left leg was painful and lacerations of the fingers at woodwork produced pain. However, despite this apparent recovery of pain sensibility, burns on both shoulders were sustained at eighteen by leaning against a radiator. At the age of twenty-five, pin prick, vein puncture, wound dressing and aspiration of fluid collections were painful.

Neuropathic joints-Neuropathic weight-bearing joints are a feature of all reported cases of this syndrome. Like the patient described by Van der Houwen (1961) who developed neuropathic arthritis of the elbow after a fracture of the ulna, this patient developed a neuropathic elbow after a fractured head of radius.

Radiological changes were first noted in the right ankle at three years. The changes, described as inflammatory osteochondritis, involved the articular surface of the talus. Involvement of the hip joints followed. The right knee and left ankle remained normal. The degenerative changes in the joint surfaces began while the patient was indifferent to pain and progressed despite the apparent recovery of pain sensibility of the skin. The initial improvement in the right ankle predicted on radiological evidence at six years failed to continue and at twenty-five years this joint showed marked osteoarthritis (Figs. 2 to 4).

The severe progressive disorganisation of the hip and elbow joints is comparable to that found in tabes and syringomyelia, in which pain sensation is irrecoverable. All the neuropathic joints retain a full range of movement. Joint position sense is normal.

Late manifestations of the neuropathic joints were the large cystic lesions associated with both hips and the

R. P. Drummond, F.R.C.S.(Eng.), 42 Allwyn Road, Strathfield, Australia 2135.

G. K. Rose, F.R.C.S.(Eng.), Consultant Orthopaedic Surgeon, Royal Salop Infirmary, Shrewsbury, England. 
left knee. Attention was first attracted to the affected joints by local pain, and on examination showed an irregular soft fluctuant swelling associated with the joint, from which synovial fluid was aspirated repeatedly. At twenty-two, a soft swelling resembling an inguinal hernia appeared above the middle of the inguinal ligament. Arthrography showed a large cyst extending from the hip

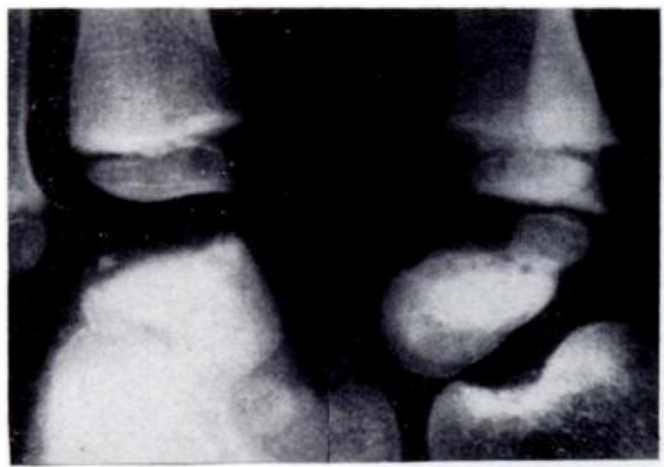

FIG. 2

Osteochondritis of the right talus at 3 years.

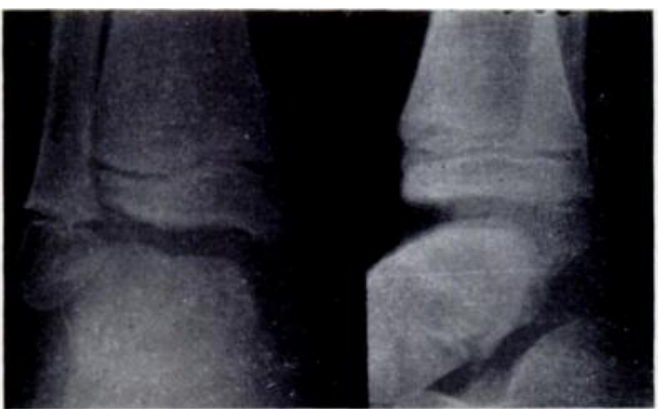

FIG. 3

Initial radiological improvement in the right ankle three years later.

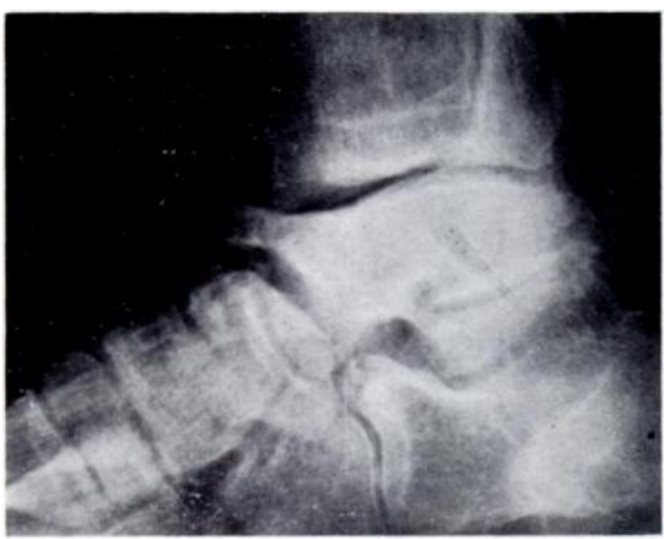

FIG. 4

Osteoarthritis of the right ankle at 25 years.

proximally beneath the left inguinal ligament to the diaphragm (Figs. 5 and 6). At operation the cyst was found to have a wall of dense fibrous tissue adherent to local structures including the femoral artery. The lining was ragged granulation tissue. No attempt was made to excise the cyst but the lining was curetted. Repeated accumulations of serous fluid in the thigh wound followed, and only after further curettage and local excision of the cyst below the inguinal ligament was healing promoted.

Histology confirmed the macroscopic appearance of acute inflammatory granulation tissue and congested oedematous fibrous tissue. Similar granulation tissue was found by Petrie (1953) in relation to a degenerative lesion of the thoracic spine with cord compression.

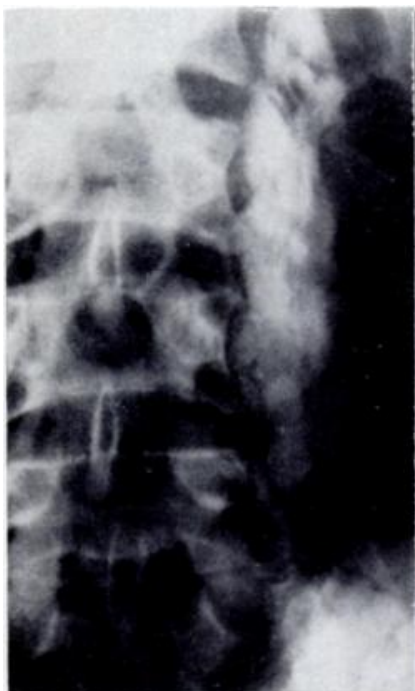

Fig. 5

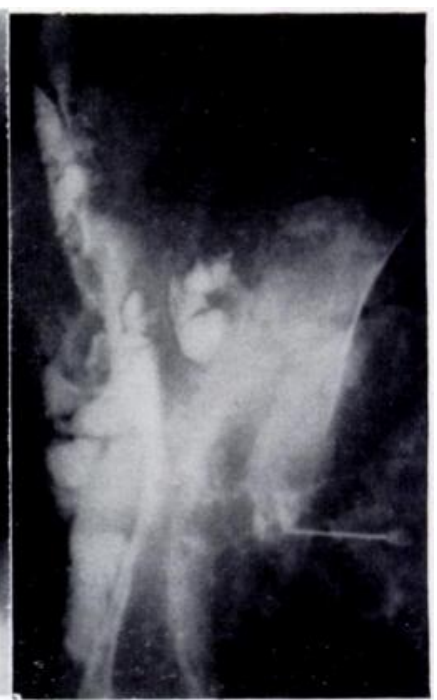

FIG. 6
Figures 5 and 6-The cystic lesion from the left hip extending proximally to the diaphragm.

The lesion associated with the right hip, excised in 1972, had a more sinister appearance at operation, consisting of poorly organised oedematous fibrous tissue. Again this was adherent to local structures and difficult to dissect free. Histologically it was composed of fibrous tissue, foreign body giant cells and spicules of bone, suggesting that these lesions represent attempts at "mopping up" the constant supply of bone eroded from the roughened joint surfaces. The large size is possibly caused by dissection along tissue planes by synovial fluid under pressure.

Osteomyelitis-The recurring osteomyelitis in congenital indifference to pain described by Murray (1957) was demonstrated in this case at fifteen years by involvement of the left radius and later of the fifth metatarsal bone. The radial osteomyelitis was at first mistaken for a Colles's fracture because the wrist was painful and swollen. Decompression of the bulging periosteum yielded a small amount of thin pus, from which staphylococcus aureus was cultured. Radiographs a month after operation showed the distal radial epiphysis to be tilted forwards but there was no progression of bone destruction. At twenty-five years a prominent ulnar styloid was the only residual deformity.

Four months after the radial osteomyelitis a discharging sinus in the sole of right foot under the fifth metatarsal head became evident. Radiographs showed osteomyclitis of the fifth metatarsal bone with sequestration of the metatarsal head. Despite sequestrectomy the 
sinus continued to discharge, and a second operation was required two years later to promote healing (Fig. 7).

Osteomyelitis could have been a manifestation of reduced resistance to infections, both bacterial and viral, for this patient had had an impressive history of childhood

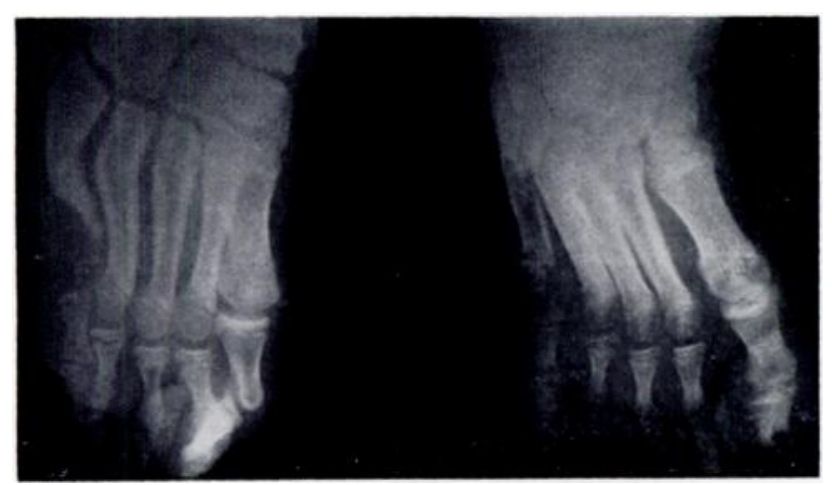

Fig. 7

Sequestration of head of fifth m.etatarsal bone from osteomyelitis.

infections. The gamma-globulin level, lymphocyte and neutrophil counts were normal.

Mucous membranes-Repeated laceration of the tongue and lips produced scarring. Subsequent scar contracture resulted in the formation of a microstoma and an irregularly shaped tongue (Fig. 8).

Fractures-Fractures were not a troublesome feature of this case. The only ununited fractures were those of the

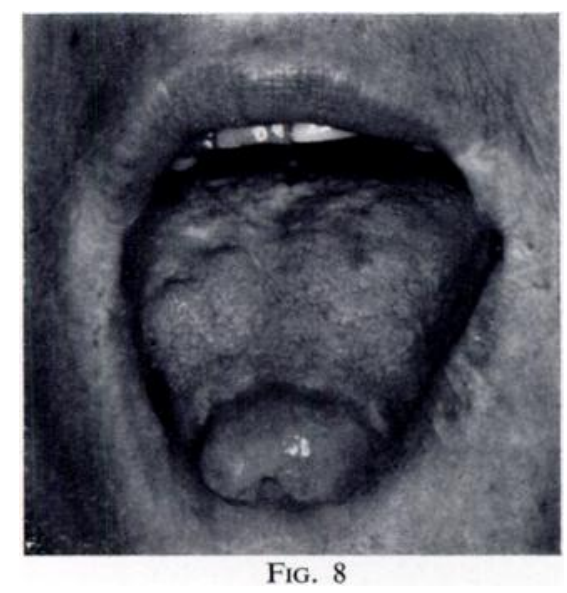

Microstoma and scarred tongue.

right fourth and fifth metatarsal bones at the age of eleven years. A fracture of the clavicle at four years united normally. As noted already, a fracture of the head of the right radius progressed to dissolution of the head and then to neuropathic arthritis of the elbow.
Last illness-The final admission to hospital was in December 1972. He then gave a three weeks' history of diarrhoea and loss of weight. There was no evidence of infection and steatorrhoea was suspected until a rectal biopsy on the day before death (January 1, 1973) showed amyloid disease. The immediate cause of death was haemorrhagic broncho-pneumonia due to a Klebsiella species. Necropsy-The kidneys were large and pale as in the nephrotic syndrome and showed extensive amyloidosis. The liver and spleen were not enlarged, but contained amyloid deposits, as did the adrenals, small and large intestine and the blood vessels of the heart, pancreas and thyroid.

The terminal diarrhoea was clearly due to amyloidosis of the intestine and the pattern of the amyloidosis suggested that it was of a secondary type. Although he had had osteomyelitis from time to time, the last occasion was eight years previously, and there was no sign of any existing infective bone lesion. It appears, therefore, that the long-standing tissue destruction was the primary cause in this case.

Both hip joints showed advanced neuropathic changes. The left contained several loose bodies up to 3 centimetres in diameter and an excess of synovial fluid. The head of the right femur was completely disarticulated: there was a false joint and the cavity contained bloodstained fluid with pieces of necrotic tissue in it.

The brain and spinal cord were sent to Dr G. W. Pearce for examination at the Midland Centre for Neurosurgery and Neurology, Smethwick. He reported that the posterior roots in their terminal millimetre or so still attached to the posterior surface of the cord suggested some loss of the larger myelinated nerve fibres and a similar slight loss was also suggested in the posterior columns of the cord, but no defect was found in the posterior column nuclei. These findings favour some form of chronic sensory neuropathy and do not appear to support a diagnosis of congenital insensitivity to pain where the lesion is probably in the upper part of the brain stem or related areas and might well be physiological in nature. The changes found in this brain were slight and non-specific.

The disturbed physiology and neuropathology of congenital and chronic sensory neuropathy and of congenital insensitivity to pain remain a very poorly understood field of neurology, and it would be an advantage in future necropsy examinations if care were taken to examine, in addition to brain and spinal cord, posterior nerve root ganglia, sensory nerves, skin and joints.

Our thanks are due to Dr Michael Symons, Consultant Histopathologist at the Royal Salop Infirmary, and to Dr G. W. Pearce, Consultant Neuropathologist, The Midland Centre for Neurosurgery and Neurology, Smethwick, Staffordshire.

\section{REFERENCES}

Houwen, H. van der (1961) A case of neuropathic arthritis caused by indifference to pain. Journal of Bone and Joint Surgery, 43-B, $314-317$. Murray, R. O. (1957) Congenital indifference to pain with special reference to skeletal changes. British Journal of Radiology, N.S. 30, 2-6. Petrie, J. G. (1953) A case of progressive joint disorders caused by insensitivity to pain. Journal of Bone and Joint Surgery, 35-B, 399-401. Rose, G. K. (1953) Arthropathy of the ankle in congenital indifference to pain. Journal of Bone and Joint Surgery, 35-B, 408-410. 